\title{
Correction to: Tryptophan depletion under conditions that imitate insulin resistance enhances fatty acid oxidation and induces endothelial dysfunction through reactive oxygen species-dependent and independent pathways
}

Theodoros Eleftheriadis ${ }^{1}$ (1) - Georgios Pissas ${ }^{1}$ - Maria Sounidaki ${ }^{1}$. Georgia Antoniadi ${ }^{1}$. Christos Rountas ${ }^{2}$. Vassilios Liakopoulos ${ }^{1} \cdot$ Ioannis Stefanidis ${ }^{1}$

Published online: 24 January 2018

(c) Springer Science+Business Media, LLC, part of Springer Nature 2018

\section{Correction to: Mol Cell Biochem (2017) 428:41-56 \\ https://doi.org/10.1007/s11010-016-2915-7}

In the original publication of the article, last author's name was misspelt. The correct name is given here.

The original article can be found online at https://doi.org/10.1007/ s11010-016-2915-7.

Theodoros Eleftheriadis

teleftheriadis@yahoo.com

1 Department of Nephrology, Medical School, University of Thessaly, Neo Ktirio, Mezourlo Hill, 41110 Larissa, Greece

2 Department of Interventional Radiology, Medical School, University of Thessaly, Larissa, Greece 